\title{
Ameloblastoma mandibular maligno con metástasis hepática y pulmonar: Caso clínico*
}

\author{
Drs. RICARDO YÁÑEZ M. ${ }^{1,}$, CRISTIAN GAMBOA C. ${ }^{1}$, JORGE MARTÍNEZ C. ${ }^{1}$, ERIC ORELLANA U. ${ }^{2}$, \\ JOSÉ M. CLAVERO R. ${ }^{3}$, IGNACIO GOÑI E. ${ }^{4}$, JAVIERA TORRES M. ${ }^{5}$, NICOLÁS JARUFE C. ${ }^{1}$ \\ Departamento Cirugía Digestiva, División de Cirugía. \\ 2 Centro del Cáncer. \\ 3 Departamento de Cirugía de Tórax. \\ 4 Departamento de Cirugía Oncológica. \\ 5 Departamento de Anatomía Patológica. \\ Facultad de Medicina, Pontificia Universidad Católica de Chile. Santiago, Chile.
}

\begin{abstract}
Malignant mandibular ameloblastoma with hepatic and lung metastases: a case report

Ameloblastoma is a benign rare tumor, originated in the odontogenic epithelium, is locally invasive and with high tendency to local recurrence, a variety less common is the malignant or metastasizing ameloblastoma, where more frequent sites of deployment are the lung and cervical lymph nodes, being much less frequent sites the liver and skull among others. The surgical treatment is mainly the aggressive surgery of the primary tumor and metastases. We present a case of a patient with malignant mandibular ameloblastoma with metastases in liver and lung, which was subjected to a subtotal mandibulectomy, local radiotherapy and lung and liver metastasectomy in a second time, with late recurrence of the lesion.
\end{abstract}

Key words: Ameloblastoma, metastases, hepatectomy.

\section{Resumen}

El ameloblastoma es un tumor poco frecuente, benigno, originado en el epitelio odontogénico, es localmente invasivo y con alta tendencia a la recurrencia local, una variedad mucho menos frecuente es el ameloblastoma maligno o que causa metástasis, donde los sitios más frecuentes de implantación son el pulmón y los linfonodos cervicales, siendo sitios mucho menos frecuentes hígado y cráneo, entre otros. El tratamiento es principalmente quirúrgico con cirugía agresiva del tumor primario y posteriormente de las metástasis. Se presenta el caso de un paciente portador de ameloblastoma mandibular maligno, con metástasis hepáticas y pulmonares, que fue sometido a una mandibulectomía subtotal y radioterapia local. Posteriormente, resección de metástasis pulmonares y hepáticas en un segundo tiempo, con recidiva de las lesiones.

Palabras clave: Ameloblastoma, metástasis, hepatectomía.

*Recibido el 22 de Diciembre de 2008 y aceptado para publicación el 19 de Febrero de 2009.

Correspondencia: Dr. Ricardo Yáñez M.

Marcoleta 352, Santiago, Chile. Fax 5623543462

E-mail: ryanezm@puc.cl 


\section{Introducción}

El ameloblastoma en una neoplasia benigna poco frecuente, originada del epitelio odontogénico, localmente agresiva, con alta tendencia a la recurrencia local de no ser removida adecuadamente, de etiología desconocida. Representa el $10 \%$ de los tumores maxilares y mandibulares, afectando por igual a hombres y mujeres, siendo la edad media de presentación a los 35 años ${ }^{1,2}$. El $80 \%$ de los ameloblastomas se origina en la mandíbula, cercano a la región molar y ramas mandibulares y la sintomatología puede incluir aumento de volumen submucoso de crecimiento lento, pérdida de piezas dentarias, mal oclusión, parestesias y dolor localizado $^{3}$.

Con mucho menos frecuencia el ameloblastoma puede presentar evolución maligna y desarrollar diseminación hematógena ${ }^{3,4}$. La variedad maligna o que origina metástasis sólo se distingue del ameloblastoma benigno por la presencia de metástasis, sin características histológicas específicas ${ }^{5}$. Los sitios más frecuentes de implante de metástasis son los pulmones $(75 \%)$, seguido de linfonodos cervicales y espinales (15\%), otras localizaciones menos frecuentes como hígado, cráneo, cerebro, riñón e intestino delgado también han sido comunicadas, pero con mucho menor incidencia ${ }^{5,6}$. Por último, otra variedad la constituye el carcinoma ameloblástico, que presenta características citológicas tales como atipia en combinación con las que presenta el ameloblastoma, independiente de la presencia de metástasis, existiendo a su vez dos subtipos, el carcinoma ameloblástico primario y el secundario o desdiferenciado el cual se desarrolla a partir de un ameloblastoma benigno preexistente ${ }^{7}$.

En base a lo expuesto se presenta el caso de un paciente con ameloblastoma maligno con metástasis hepáticas y pulmonares tratado en nuestro centro.

\section{Caso clínico}

Se presenta el caso de un paciente varón de 50 años de edad, sin antecedentes mórbidos de importancia, con cuadro de seis semanas de evolución caracterizado por dolor dental y de mandíbula inferior con respuesta parcial al paracetamol. Consulta a odontólogo quien solicita radiografía dental, donde se observa imagen radiolúcida extensa, unilocular de límites poco definidos con destrucción de tabla ósea. Se realiza biopsia por punción de la lesión que muestra ameloblastoma. En estudio de diseminación, se realiza una tomografía axial computada (TAC) de cabeza, cuello y tórax que muestra lesión lítica mandibular con compromiso de partes blandas y extensión al piso de la boca, múltiples nódulos pulmonares bilaterales y en base derecha, con características de localizaciones secundarias, y lesión focal hepática en lóbulo izquierdo de $5,8 \times 6,9 \mathrm{~cm}$ que puede corresponder a lesión secundaria. Cintigrama muestra actividad osteoblástica anormal a nivel del mentón. Continuando el estudio, se realiza punción hepática bajo TAC cuya biopsia es compatible con ameloblastoma, siendo entonces el tumor primario de origen dental con metástasis pulmonares y hepáticas.

El caso es discutido en reunión multidisciplinaria y dado el carácter benigno del tumor y su lento crecimiento, se decide someter al paciente a resección del tumor primario y metástasis en distintos tiempos, a la vez que se inicia quimioterapia con Taxol y Carboplatino en seis ciclos, logrando estabilización de las lesiones. Se efectúa hemimandibulectomía inferior con reconstrucción con colgajo deltopectoral e injerto microvascular del peroné (Figura 1). El paciente debe ser sometido posteriormente a aseo quirúrgico y colgajo por dehiscencia del piso de la boca, evolucionando en buenas condiciones.

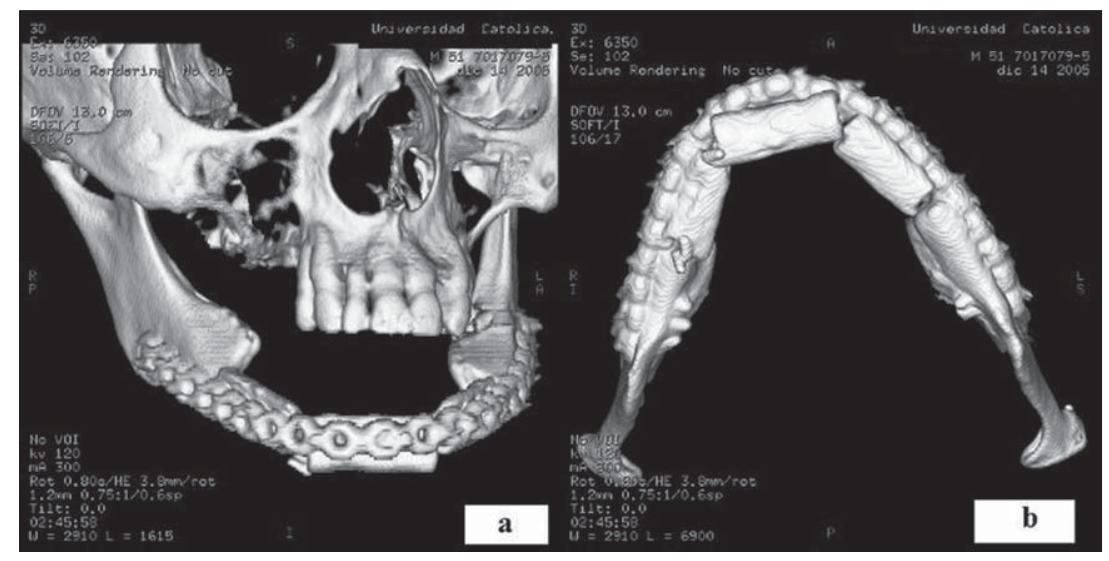

Rev. Chilena de Cirugía. Vol 61 - N 5, Octubre 2009; pág. 458-462
Figura 1. TAC de cabeza con reconstrucción tridimensional; a, b: Visión latero-frontal e inferior, respectivamente, de la reconstrucción mandibular realizada en la extracción 
En la biopsia se observa un ameloblastoma maligno variedad folicular de 4 × 2,5 x 2,5 cm con extensas zonas de infiltración perineural y destrucción ósea del cuerpo de la mandíbula (Figura 2).

A los dos meses se realiza hepatectomía izquierda extendida (segmentos I, II, II, IV, V, VIII), donde no se observa diseminación peritoneal y la exploración hepática y ecografía intraoperatoria demuestran lesión de $10 \mathrm{~cm}$ de diámetro en lóbulo hepático izquierdo sin compromiso de la vena hepática derecha y compresión de la vena hepática media. La pieza operatoria muestra a la histología metástasis de ameloblastoma de $10 \times 8 \times 7 \mathrm{~cm}$ que alcanza focalmente el borde quirúrgico entintado (Figuras 3 y 4).

El paciente tiene una recuperación satisfactoria y posteriormente se realiza resección de metástasis pulmonar, quedando sin evidencia de enfermedad, manteniendo capacidad funcional de $100 \%$. Se realiza radioterapia en zona mandibular focalmente en lecho quirúrgico usando radioterapia de intensidad modulada (IMRT).

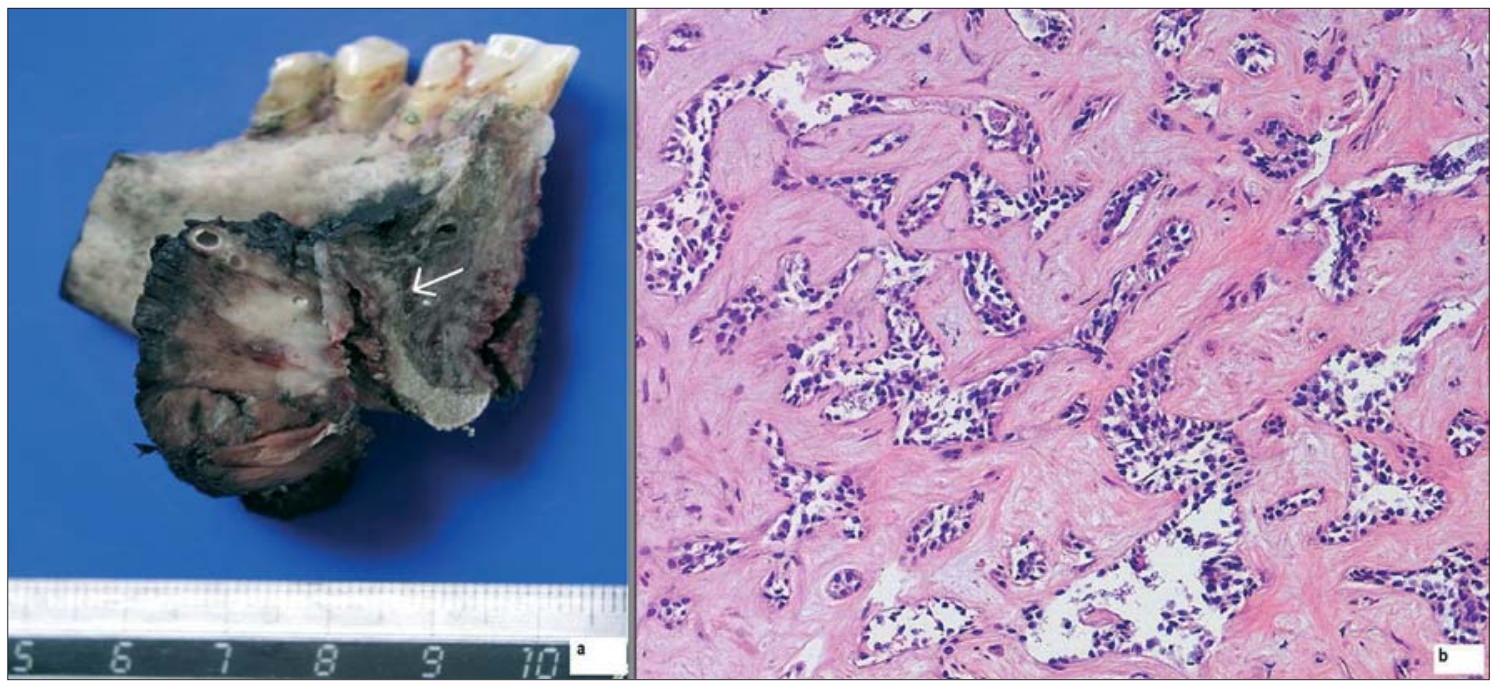

Figura 2. a: Hemimandibulectomía inferior que muestra lesión tumoral blanco grisácea, de bordes mal delimitados, que destruye el hueso (cuerpo mandibular) e infiltra el tejido muscular esquelético. b: Ameloblastoma maligno variedad folicular, con extensas zonas de infltración perineural y destrucción ósea del cuerpo de la mandíbula.
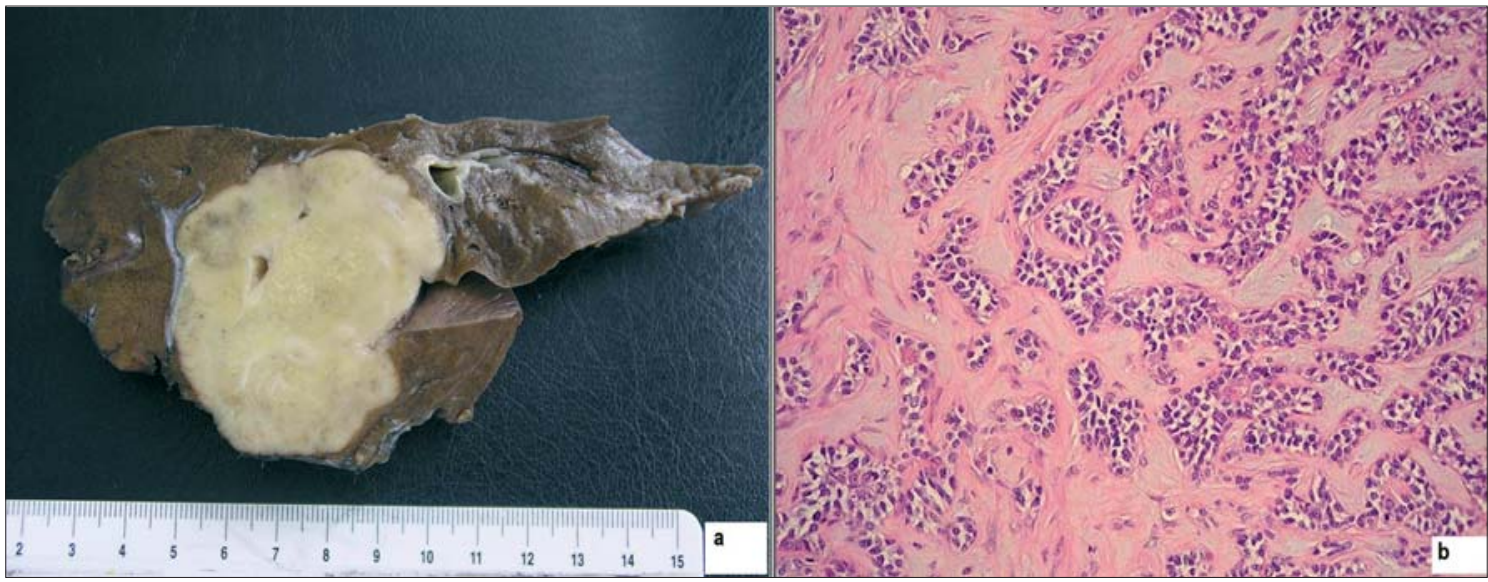

Figura 3. a: Lóbulo hepático izquierdo de 550 gr donde se reconoce tumor blanquecino amarillento, de bordes polilobulados, bien delimitados, de consistencia dura, que mide $10 \times 8 \times 7 \mathrm{~cm}$ y que alcanza focalmente el borde entintado. b: Ameloblastoma de las mismas características que tumor primario mandibular. 


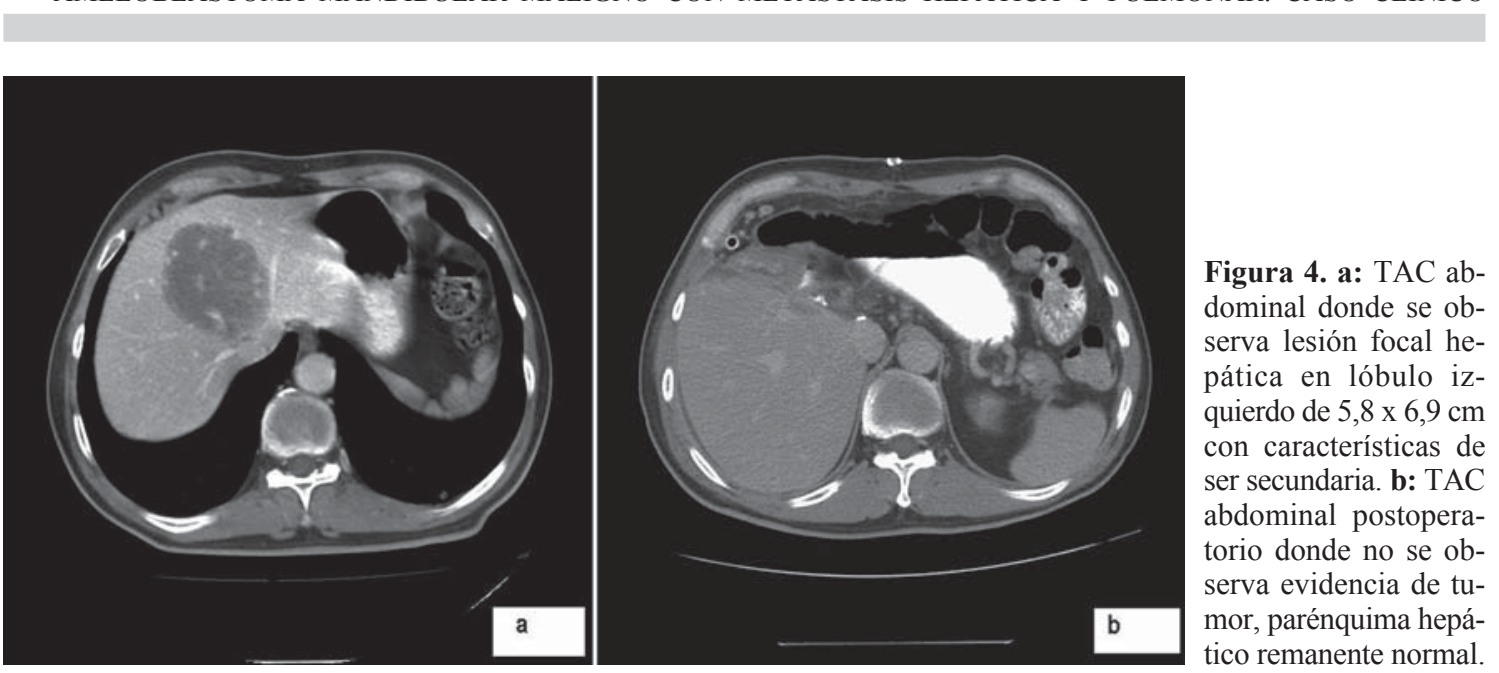

Luego de tres meses se realiza TAC donde se observa nuevas lesiones hepáticas y nódulos pulmonares. Se discute el caso y se considera irresecable, planteándose alternativa de nueva quimioterapia en vista de las buenas condiciones del paciente y agresividad de tumor, iniciándose con seis ciclos al igual que la anterior, logrando estabilización de la enfermedad.

\section{Discusión}

Aunque los ameloblastomas usualmente progresan en forma lenta, si son localmente invasivos y no tratados pueden causar morbilidad significativa y hasta ser de riesgo vital, más aún en los casos metastásicos como el presentado ${ }^{8}$.

La variedad maligna (que causa metástasis) es extremadamente poco frecuente, con una incidencia de $1-2 \%$ de todos los ameloblastomas, la edad media de presentación es de 34,4 años, con un rango de 5-74 años, con sobrevida media de tres meses a cinco años desde que es diagnosticada la metástasis, siendo de 25 años la mayor sobrevida reportada ${ }^{4,9}$.

Según la última clasificación de la WHO Cabeza y Cuello, 2005, se aceptan entre los Tumores Odontogénicos malignos cuatro tipos distintos de Carcinomas Odontogénicos Ameloblásticos (Ameloblastomas malignos) $)^{2,3,10}$ :

a) Ameloblastoma que causa metástasis: corresponde a un ameloblastoma que origina metástasis y tiene aspecto microscópico benigno similar al Ameloblastoma benigno clásico, tanto en el primario como en las metástasis.

b) Carcinoma Ameloblástico tipo primario: comparte comportamiento primario con su aspecto microscópico que presenta criterios morfológicos celulares concluyentes para establecer malignidad, tanto en el primario como en las metástasis.

c) Carcinoma ameloblástico tipo secundario (desdiferenciado), intraóseo: ameloblastoma previamente benigno y que en sus metástasis presenta atipías celulares.

d) Carcinoma ameloblástico tipo secundario (desdiferenciado), periférico: transformación de un Ameloblastoma periférico preexistente que se malignizó.

El diagnóstico es establecido en base al examen físico, incluyendo examen de cabeza y cuello, la evaluación radiológica incluye pantomografía. Los ameloblastomas se presentan radiológicamente como una lesión radiolúcida bien definida uni o multilocular rodeada de un borde opaco. Comúnmente el tumor es más grande de lo que se aprecia en la radiografía ${ }^{11,12}$.

Varios factores han sido asociados con el mayor riesgo del desarrollo de metástasis, las que ocurren en casos de larga evolución, recurrencias locales múltiples, procedimientos quirúrgicos numerosos o radioterapia, sin embargo, no todos los ameloblastomas metastásicos tienen historial de intervenciones quirúrgicas a repetición tal como es el caso presentado. También han sido asociados con el desarrollo de metástasis la extensión local del tumor y la localización mandibular del primario ${ }^{1,4,11}$.

Ya que existe un gran intervalo entre el diagnóstico del tumor primario y la ocurrencia de la metástasis, ya sea regional o a distancia, que en promedio es de 10 a 12 años, se recomienda al menos una radiografía de tórax al año a modo de seguimiento ya que es el sitio más frecuente de metástasis a distancia $^{3,13}$. 
El elemento más importante en el manejo del ameloblastoma metastásico es el tratamiento de la lesión primaria. Como fue mencionado previamente, las cirugías múltiples aumentan significativamente el riesgo de metástasis. Con respecto al potencial de recurrencia y metástasis, el curetaje del tumor primario lleva el peor de los pronósticos, obteniéndose mucho mejores resultados con la resección tumoral con márgenes de tejido sano de al menos 1 a $1,5 \mathrm{~cm}^{13,14}$.

Distintas alternativas para el tratamiento de las metástasis pulmonares y hepáticas son utilizadas, siendo la quirúrgica la más frecuente, sin embargo, la quimioterapia y radioterapia también han sido descritas, donde las revisiones actuales recomiendan estas últimas para cuidados paliativos y el manejo quirúrgico agresivo para lesiones tratables ${ }^{15}$. La terapia quirúrgica consiste en resección amplia, cuya extensión dependerá del número y localización de las lesiones, si fuese necesario contar con diagnóstico histológico preoperatorio es posible realizarlo por punción con aguja fina guiada por $\mathrm{TAC}^{14-16}$.

Para aquellas lesiones fuera de alcance quirúrgico, la radiación y la quimioterapia son las únicas opciones existentes actualmente, sin embargo, se ha visto que la radioterapia presenta resultados impredecibles y una alta tasa de recurrencia, siendo utilizada en casos específicos por algunos autores, tales como extensos tumores primarios maxilares ${ }^{15,16}$.

La quimioterapia, sin llegar a ser curativa, tiene un efecto paliativo en la sintomatología de los pacientes y en algunos casos se ha asociado a reducción del tamaño tumoral, siendo beneficiosa cuando las lesiones son extensas o están en relación a estructuras vitales y en casos paliativos en que existen lesiones inoperables ${ }^{16,17}$.

Existiendo pocos casos de metástasis hepática reportados, es necesario contar con un mayor número para lograr una aproximación terapéutica más adecuada.

\section{Referencias}

1. Mendenhall WM, Werning JW, Fernandes R, Malyapa RS, Mendenhall NP. Ameloblastoma. Am J Oncology 2007; 30: 645-648.

2. Reichart PA, Philipsen HP, Sonner S. Ameloblastoma: biological profile of 3.677 cases. Eur J Cancer B Oral Oncol 1995; 31: 86-99.

3. Kim SG, Jang HS. Ameloblastoma: a clinical, radiographic, and histopathologic analysis of 71 cases. Oral
Surg Oral Med Oral Pathol Oral Radiol Endod 2001; 91: 649-653.

4. Ladeinde AL, Ogunlewe MO, Bamgbose BO, Adeyemo WL, Ajayi OF, Arotiba GT, et al. Ameloblastoma: analysis of 207 cases in a Nigerian teaching hospital. Quintessence Int 2006; 37: 69-74.

5. Gilijamse M, Leemans CR, Winters HA, Schulten EA, van der Waal I. Metastasizing ameloblastoma. Int J Oral Maxillofacial Surg 2007; 36: 462-464.

6. Laughlin EH. Metastasizing ameloblastoma. Cancer 1989; 64: 776-780.

7. Barnes L, Eveson JW, Reichart P, Sidransky D, eds: World Health Organization Classification of Tumours. Pathology an Genetics. Head and Neck Tumours. Lyon: International Agency for Research on Cancer (IARC). IARC Press 2005.

8. Zwahlen RA, Grätz KW. Maxillary ameloblastomas: a review of the literature and of a 15 -year database. J Craniomaxillofac Sug 2002; 30: 273-279.

9. Vallicioni J, Loum B, Dassonville O, Poissonnet G, Ettore F, Demard F. Ameloblastomas. Ann Otolaryngol Chir Cervicofac 2007; 124: 166-171.

10. Mathew S, Rappaport K, Ali SZ, Busseniers AE, Rosenthal DL. Ameloblastoma. Cytologic findings and literature review. Acta Cytol 1997; 41: 955-960.

11. Becelli R, Carboni A, Cerulli G, Perugini M, Iannetti G. Mandibular ameloblastoma: analysis of surgical treatment carried out in 60 patients between 1977 and 1998. J Craniofac Surg 2002; 13: 395-400.

12. Zemann W, Feichtinger M, Kowatsch E, Kärcher H. Extensive ameloblastoma of the jaws: surgical management and immediate reconstruction using microvascular flaps. Oral Surg Oral Med Oral Pathol Oral Radiol Endol 2007; 103: 190-196.

13. Sampson DE, Pogrel MA. Management of mandibular ameloblastoma: the clinical basis for a treatment algorithm. J Ora1 Maxillofac Surg 1999; 57: 10741077.

14. Ueda M, Kosaki K, Kaneda T, Imaizumi M, Abe T. Doubling time of ameloblastoma metastasizing to the lung: report of two cases. J Craniomaxillofac Surg 1992; 20: $320-322$.

15. Ciment LM, Ciment AJ. Malignant ameloblastoma metastatic to the lungs 29 years after primary resection: a case report. Chest 2002; 121: 1359-1361.

16. Newman L, Howells GL, Coghlan KM, DiBiase A, Williams DM. Malignant ameloblastoma revisited. $\mathrm{Br}$ J Oral Maxillofac Surg 1995; 33: 47-50.

17. Senra GS, Pereira AC, Murilo dos Santos L, Carvalho YR, Brandão AA. Malignant ameloblastoma metastasis to the lung: a case report. Oral Surg Oral Med Oral Pathol Oral Radiol Endod 2008; 105: e42-46. 